\title{
Association nirmatrelvir/ritonavir contre la COVID-19
}

\author{
Emily G. McDonald MD MSc, Todd C. Lee MD MPH
}

Citation : CMAJ 2022 February 14;194:E218. doi : 10.1503/cmaj.220081-f

Voir la version anglaise de l'article ici : www.cmaj.ca/lookup/doi/10.1503/cmaj.220081

\begin{abstract}
1 L'association nirmatrelvir/ritonavir (Paxlovid) est un médicament antiviral administré par voie orale et approuvé par Santé Canada pour contrer l'activité du SRAS-CoV-2

Ce traitement est indiqué chez les patients ambulatoires adultes ( $\geq 18$ ans) atteints d'une forme non hypoxique de la COVID-19 exposés à un risque élevé de progression de leur maladie vers une forme sévère (p. ex., les personnes d'un âge avancé, qui ont des comorbidités, qui ne sont pas vaccinées ou qui sont immunodéprimées) ${ }^{1,2}$.
\end{abstract}

2

Les études, pour lesquelles on a recruté

principalement des participants non vaccinés, se sont déroulées avant l'apparition du variant Omicron et n'ont pas encore été révisées par des pairs

Selon les données disponibles, les patients présentant un risque d'hospitalisation de $5 \%$ sont associés à un nombre estimé de sujets à traiter pour prévenir 1 hospitalisation de 24 (intervalle de confiance à $95 \% 22-29)^{3,4}$. Les effets indésirables les plus fréquents étaient la dysgueusie, la diarrhée, des vomissements, une hausse de la tension artérielle et des maux de tête.

Le traitement se présente dans un emballage intégrant le nirmatrelvir $(300 \mathrm{mg}-2$ comprimés à $150 \mathrm{mg}$ ) et le ritonavir ( 1 comprimé à $100 \mathrm{mg}$ ); les 3 comprimés sont pris ensemble, 2 fois par jour pendant 5 jours

Le traitement doit être instauré dès que possible après la confirmation d'un diagnostic de COVID-19, idéalement dans les 5 jours suivant l'apparition des symptômes. Bien que des données d'observation sur l'innocuité du ritonavir pendant la grossesse existent, ce n'est pas le cas pour le nirmatrelvir. Dans le cas d'une insuffisance rénale modérée (taux de filtration glomérulaire estimé [TFGe] de $30-60 \mathrm{~mL} / \mathrm{min}$ ), la dose est diminuée à 1 comprimé de nirmatrelvir et 1 comprimé de ritonavir, administrés 2 fois par jour. Le nirmatrelvir est contre-indiqué si le TFGe $<30 \mathrm{~mL} / \mathrm{min}$.

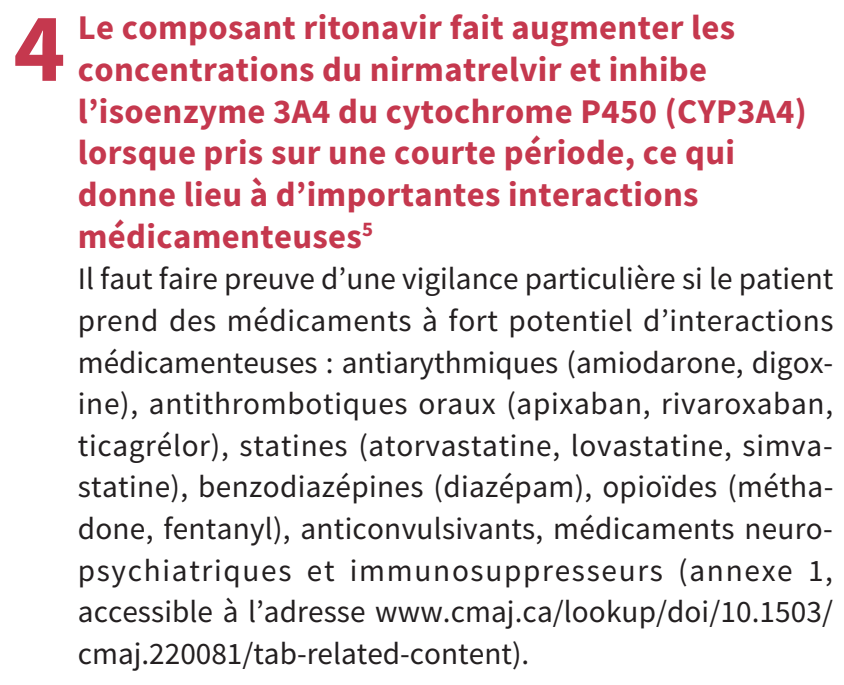

5

Les mesures d'atténuation des interactions médicamenteuses comprennent les réductions de dose, la substitution ou l'interruption temporaire d'un médicament et la surveillance du traitement médicamenteux

De telles mesures doivent être instaurées pendant le traitement et jusqu'à $3-5$ jours après celui-ci ${ }^{5}$. Certains médicaments (voir annexe 1) diminuent l'efficacité de l'association nirmatrelvir/ritonavir et peuvent mener à l'échec du traitement ou à une résistance virologique ${ }^{1}$; on doit alors envisager d'autres traitements contre la COVID-194. Dans de nombreux cas, on recommande la consultation d'un pharmacien (annexe 1).

\section{Références}

1. The COVID-19 Treatment Guidelines Panel's statement on potential drug-drug interactions between ritonavir-boosted nirmatrelvir (Paxlovid) and concomitant medications. Bethesda (MD): National Institutes of Health; updated 2021 Dec. 30. Accessible ici : https://www.covid19treatmentguidelines.nih.gov/therapies/ statement-on-paxlovid-drug-drug-interactions/ (consulté le 16 janv. 2022).

2. Product monograph including patient medication information: Paxlovid. Kirkland (QC): Pfizer Canada ULC; 2022. Accessible ici : https://pdf.hres.ca/ dpd_pm/00064313.PDF (consulté le 23 janv. 2022). 
3. Pfizer announces additional phase $2 / 3$ study results confirming robust efficacy of novel COVID-19 oral antiviral treatment candidate in reducing risk of hospitalization or death. New York: Pfizer; 2021. Accessible ici : https://www.pfizer.com/news/press -release/press-release-detail/pfizer-announces-additional-phase-23-study-results (consulté le 17 janv. 2022).

4. Lee TC, Morris AM, Grover SA, et al. Outpatient therapies for COVID-19: How do we choose? Open Forum Infect Dis 2022 Jan. 19 [Cyberpublication avant l'impression]. doi: 10.1093/ofid/ofac008.

5. Fact sheet for healthcare providers: emergency use authorization for Paxlovid. Silver Spring (MD): US Food and Drug Administration; revised 2021 Dec. 22. Accessible ici : https://www.fda.gov/media/155050/download (consulté le 16 janv. 2022).

Intérêts concurrents : Emily McDonald déclare recevoir une aide salariale du Fonds de recherche du Québec - Santé (FRQS) et des fonds de fonctionnement des Instituts de recherche en santé du Canada (IRSC). La Dre McDonald détient aussi des droits d'auteur du logiciel MedSécure. Todd Lee déclare recevoir une aide salariale de recherche du FRQS et des fonds de fonctionnement des IRSC et de l'Initiative interdisciplinaire en infection et immunité de l'Université McGill (MI4). Le Dr Lee détient aussi des droits d'auteur du logiciel MedSécure.

Cet article a été révisé par des pairs.

Affiliations : Unité d'évaluation des pratiques cliniques (McDonald, Lee) et divisions de médecine interne (McDonald) et d'infectiologie (Lee), Département de médecine, Centre universitaire de santé McGill, Montréal, Que.

Propriété intellectuelle du contenu : Il s'agit d'un article en libre accès distribué conformément aux modalités de la licence Creative Commons Attribution (CC BY-NC-ND 4.0), qui permet l'utilisation, la diffusion et la reproduction dans tout médium à la condition que la publication originale soit adéquatement citée, que l'utilisation se fasse à des fins non commerciales (c.-à-d., recherche ou éducation) et qu'aucune modification ni adaptation n'y soit apportée. Voir : https:// creativecommons.org/licenses/by-nc-nd/4.0/deed.fr

Correspondance : Emily McDonald, emily.mcdonald@mcgill.ca

Le JAMC vous invite à soumettre vos textes pour la rubrique " Cinq choses à savoir ... » en ligne à http://mc.manuscriptcentral.com/cmaj. 